\title{
Chlamydia trachomatis Infection Control Programs: Lessons Learned and Implications for Vaccine Development
}

\author{
Jean M. Chavez, Rodolfo D. Vicetti Miguel, and Thomas L. Cherpes \\ Department of Pediatrics, University of Pittsburgh School of Medicine, Rangos Research Center, Room 9123, \\ 4401 Penn Avenue, Pittsburgh, PA 15224, USA \\ Correspondence should be addressed to Thomas L. Cherpes, cherpestl@upmc.edu
}

Received 15 July 2011; Accepted 29 August 2011

Academic Editor: Richard L. Sweet

Copyright ( $\odot 2011$ Jean M. Chavez et al. This is an open access article distributed under the Creative Commons Attribution License, which permits unrestricted use, distribution, and reproduction in any medium, provided the original work is properly cited.

Chlamydia trachomatis control efforts that enhance detection and treatment of infected women may paradoxically increase susceptibility of the population to infection. Conversely, these surveillance programs lower incidences of adverse sequelae elicited by genital tract infection (e.g., pelvic inflammatory disease and ectopic pregnancy), suggesting enhanced identification and eradication of C. trachomatis simultaneously reduces pathogen-induced upper genital tract damage and abrogates formation of protective immune responses. In this paper, we detail findings from C. trachomatis infection control programs that increase our understanding of chlamydial immunoepidemiology and discuss their implications for prophylactic vaccine design.

\section{Introduction}

An estimated 90 million individuals sexually acquire Chlamydia trachomatis infection each year (including 45 million in Asia, 15 million in Africa, and 4 million in the United States [1]), and in all these geographic regions the highest prevalences of chlamydial genital tract infection are found among adolescents and young adults [2]. Although C. trachomatis is a common cause of male nongonococcal urethritis [3], female genital tract infections represent more significant threats to reproductive health. Morbidities associated with $C$. trachomatis genital tract infections in women include pelvic inflammatory disease (PID) and its sequelae of chronic pelvic pain, ectopic pregnancy, and tubal infertility [4].

\section{Shortcomings and Benefits of Infection Control Programs}

During the past 25 years, many areas in Europe and North America implemented infection control programs to reduce sexual transmission of $C$. trachomatis [5]. These programs typically relied upon widespread screening and prompt treatment of asymptomatic individuals as a conduit for decreased population infectivity [6-8]. Applying these principles, one regional United States program reduced $C$. trachomatis prevalence $60 \%$ among young women during the first 9 years of its existence [9]. However, similar screening and treatment of young women in this region during the succeeding 7 years was associated with a $46 \%$ increase in chlamydial positivity [10]. This scenario was repeated in British Columbia-after introduction of a C. trachomatis infection control program case rates fell from 216 to 104 cases per 100000 individuals but then steadily climbed to 193 cases per 100000 individuals [11]. In fact, nearly all countries implementing large-scale chlamydial control programs have reported increased case report numbers despite ongoing control efforts [12-14], suggesting expanded earlier treatment may enhance population susceptibility to $C$. trachomatis infection. Although the higher prevalences of $C$. trachomatis seen in areas with active surveillance may have been sequelae to increased screening of higher risk women or increased use of more sensitive diagnostic tests, at least in 1 such surveillance program higher prevalence appeared to reflect actual increases in chlamydial positivity [15]. Providing stronger support for the supposition that C. trachomatis infection control programs increase population susceptibility to infection, British Columbia saw 
reinfection rates rise from 9.7 to 53.2 cases per 100,000 individuals in the midst of widespread control efforts $[11,15]$.

Concurrent with higher incidences of reinfection, however, C. trachomatis control programs have reduced genital tract complications elicited by infection. For example, concomitant with steady increases in C. trachomatis case numbers, health officials in San Francisco County, Calif, observed dramatic decreases in PID and ectopic pregnancy cases [16]. Earlier diagnosis and treatment of genital tract chlamydial infections was also associated with sharp reductions in ectopic pregnancy rates in Norway and Sweden $[17,18]$. Despite the substantially increased rates of $C$. trachomatis infection documented in British Columbia [11], surveillance data demonstrated robust decreases in annual case numbers and rates of PID, ectopic pregnancy, and tubal factor infertility [19]. Taken together, these data imply that enhanced detection and earlier treatment of infected women achieved upon implementation of C. trachomatis infection control programs may have been responsible for reduced incidences of the adverse outcomes associated with ascension of this pathogen into the upper genital tract. These data further imply that persistent $C$. trachomatis infection, not simply acquisition or reinfection, may be the scenario most likely responsible for development of PID, ectopic pregnancy, and tubal factor infertility.

\section{C. trachomatis Control Programs and the Arrested Immunity Hypothesis}

Seminal investigations performed in British Columbia allowed Brunham et al. to first posit that C. trachomatis infection control programs increase population susceptibility to reinfection [11]. Their "altered immunity" hypothesis states that development of protective immune responses against $C$. trachomatis is abrogated by earlier detection and treatment of infected individuals and further argues this interrupted development of protective immunity increases the likelihood of reinfection. Evidence supporting this proposed linkage between expanded earlier treatment and increased population susceptibility to infection has been provided by both experimental and clinical investigations. Compared to untreated mice, humoral immune responses were impaired in the vaginas of mice administered doxycycline within the first 10 days of primary intravaginal chlamydial infection. Moreover, these same antibiotic-treated mice were also less protected from chlamydial reinfection [20]. Taken together, these data suggest that accelerated eradication of chlamydia from the genital tract that was mediated by doxycycline therapy may have hampered the development of protective immune responses.

The durability of C. trachomatis infection among many women not receiving antichlamydial antibiotics implies protracted courses of infection may be needed for development of sterilizing immunity, while providing further support for the validity of the altered immunity hypothesis. For example, an annual clearance rate of $45 \%$ among asymptomatic Dutch women not receiving antimicrobial therapy suggested sterilizing immunity is often not achieved during the first year of a chlamydial genital tract infection [21]. A similar rate of clearance was seen in Colombia where 54\% (44/82) of women not receiving antichlamydial antibiotics cleared asymptomatic genital tract infection during the first year after initial diagnosis; however $C$. trachomatis infection persisted in only $6 \%$ of this cohort after 4 years of followup [22]. These results indicate the development of sterilizing immunity against genital tract chlamydial infection is most often measured in months or years, while long-term presence of the organism in the absence of overt inflammatory symptoms highlights the highly successful parasitic relationship $C$. trachomatis has achieved with its human hosts. In addition to these natural history studies, clinical data in support of the altered immune hypothesis was generated upon completion of a C. trachomatis seroprevalence study enrolling 8000 pregnant Finnish women. During the same period of time in this country in which dramatic increases in the frequency of genital tract chlamydial infection were observed [23], this study reported a $51 \%$ decrease in the prevalence of positive serum IgG antibody titers against $C$. trachomatis major outer membrane protein (MOMP) among women less than 23 years of age and a $65 \%$ decrease in positive titers among $23-$ 28-year-old women [24]. These findings may indicate that in some women chlamydial MOMP-specific humoral responses are transitory or, conversely, that humoral responses against C. trachomatis are slow to develop and that earlier identification and treatment of infection impeded the development of humoral immunity. Although more speculative, it is also possible that decreasing seroprevalence of $C$. trachomatis in Finland contributed to increased population susceptibility to reinfection. This latter clinical scenario is consistent with experimental data demonstrating that chlamydial-specific antibodies were integral for protection of female mice from genital tract reinfection [25]. Further clinical investigation, however, will be needed to determine the strength of the associations between enhanced detection and treatment, impaired humoral immune responses, and increases in susceptibility to C. trachomatis genital tract reinfection.

\section{Implications for $C$. trachomatis Vaccine Development}

Widespread C. trachomatis infection control programs reduce incidences of PID and its adverse sequelae $[19,26]$, but are associated with increased population susceptibility to infection. These seemingly contradictory observations interestingly help illuminate the immunoepidemiology of C. trachomatis infection. Although some conclusions that we draw from these epidemiological investigations remain conjectural, increased population susceptibility to chlamydial infection seen concomitantly with decreases in genital tract complications of infection indicates the following: (1) this obligate intracellular pathogen is weakly antigenic; (2) the organism requires persistent infection to elicit upper genital tract damage; (3) primary infection is associated with host immune responses that are suboptimal for immediate pathogen clearance but unlikely to damage vital upper genital tract architecture and/or this organism employs 
immunoevasion strategies that promote establishment of asymptomatic but persistent infection.

Consistent with the notion of low antigenicity, $C$. trachomatis infections of the female genital tract often remain asymptomatic. Cell walls of C. trachomatis, like other Gram-negative bacteria, contain lipopolysaccharide (LPS), a molecule that stimulates multiple responses in infected tissue including increased secretion of pro-inflammatory cytokines, macrophage activation, and increased expression of endothelial leukocyte adhesion molecules. Ex vivo assays show that LPS is primarily responsible for the increased production of tumor necrosis factor (TNF) elicited by $C$. trachomatis elementary bodies, even though chlamydia LPS is 100 -fold less potent for the production of this key proinflammatory cytokine than the LPS isolated from Neisseria species [27]. Therefore, epidemiological data suggesting that the presence of persistent genital tract infection promotes chlamydial disease expression is consistent with this ability of $C$. trachomatis to elicit less robust inflammatory responses [28]. In other words, pelvic inflammatory disease, fallopian tube scarring, and tubal infertility may more frequently result from the presence of chronic, albeit mild inflammation. Although persistent infection may be responsible for increased chlamydial disease expression, the specific immune responses that evoke this upper genital tract damage remain unknown. Mouse and nonhuman primate studies suggest IFN- $\gamma$-producing $\mathrm{CD}^{+} \mathrm{T}$ cells are needed for clearance of chlamydial infections, and the generation of appropriate type 1 immunity is often considered to be an integral component of any vaccine conferring protection against chlamydial disease expression [29]. On the other hand, increased IFN$\gamma$ production, in conjunction with other type 1 responses, may promote immunopathological responses and increase the likelihood of fallopian tube scarring and tubal factor infertility. Since genital tract $C$. trachomatis infections in nature are weakly antigenic, it is at least a theoretical concern that chlamydial vaccines that confer protection via generation of robust, more durable memory $\mathrm{T}$ cell responses could also elicit immunopathological damage if repetitive bouts of inflammation were elicited upon subsequent exposures to the organism.

C. trachomatis is known to have evolved exclusively as a human pathogen and fact which may explain why infection usually occurs in the absence of overt inflammation. Strategies developed by this intracellular pathogen to avoid host detection or clearance include replication within membranebound inclusions [30], suppression of class I and II major histocompatibility complex molecule expression by infected cells [31], and its ability to capture indole to escape IFN$\gamma$-mediated tryptophan starvation [32]. A high-frequency asymptomatic $C$. trachomatis infection, on the other hand, may be the consequence of host responses that evolved to minimize collateral damage to delicate upper genital tract structures. In support of this hypothesis, we recently saw that $C$. trachomatis genital tract infection was associated with increased numbers of endometrial CD $4^{+} \mathrm{T}$ cells, B cells, and plasma cells [33], suggesting there is a predilection for $C$. trachomatis infection to polarize endometrial inflammation toward type 2 immunity. Although type 1 immune responses

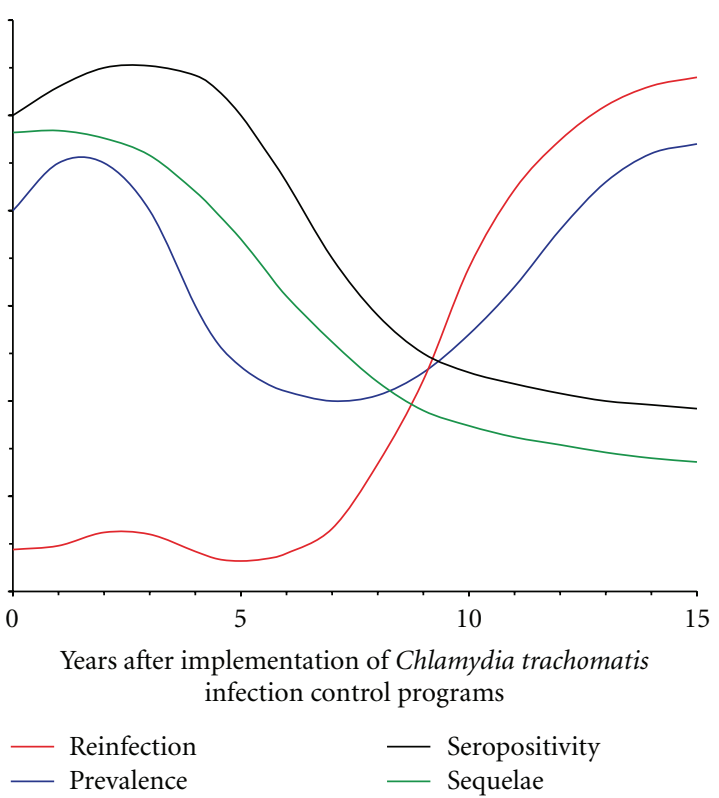

FIGURE 1: Schema summarizing outcomes associated with Chlamydia trachomatis genital tract infection control programs. These outcomes include increased number of chlamydial cases, increased rates of chlamydial reinfection, decreased detection of chlamydialspecific serum antibodies, and decreased rates of pelvic inflammatory disease, ectopic pregnancy, and tubal factor infertility. Although no causality between these observed outcomes has been established, chlamydial vaccine development will require better delineation of the linkage between enhanced early treatment and diminished antichlamydial humoral immunity, increased susceptibility to infection, and lower incidences of adverse reproductive tract sequelae.

are capable of clearing $C$. trachomatis, type 2 responses may have been selected as a safer alternative to more damaging type 1 responses in the upper genital tract [34]. Whether type 2 endometrial inflammation is associated with enhanced or impaired chlamydial clearance or higher or lower chances of immunopathological tissue damage remains unknown, but resolution of these uncertainties is essential for proper vaccine development. The reported linkage between enhanced early detection, decreased chlamydiaspecific antibody prevalence, and increased chlamydial reinfection rates does not establish causality between impaired humoral immune responses and increased susceptibility to infection, but does provide the impetus for more complete understanding of the immune responses that may impact chlamydial vaccine efficacy.

In conclusion, this brief paper summarized the findings from C. trachomatis infection control programs that alter our understanding of the immunoepidemiology of chlamydial genital tract infection (Figure 1). Although observations from these programs suggest increased duration of infection is a risk factor for the development of PID, understanding of the specific host genetic variations and immune responses that promote genital tract damage awaits further investigation. Additional work is also needed to better inform chlamydial vaccine development, as more comprehensive 
understanding of the immune responses that protect against C. trachomatis acquisition and reinfection and prevent or elicit PID development must be achieved before clinical vaccination trials can be safely initiated.

\section{Acknowledgments}

Authors acknowledge Dr. John F. Alcorn for critical paper review. Paper preparation was supported by funds provided by the National Institute of Allergy and Infectious Diseases (Grant U19AI084024).

\section{References}

[1] R. C. Brunham and J. Rey-Ladino, "Immunology of chlamydia infection: implications for a Chlamydia trachomatis vaccine," Nature Reviews Immunology, vol. 5, no. 2, pp. 149-161, 2005.

[2] Centers for Disease Control and Prevention (CDC), "CDC grand rounds: Chlamydia prevention: challenges and strategies for reducing disease burden and sequelae," Morbidity and Mortality Weekly Report, vol. 60, no. 12, pp. 370-373, 2011.

[3] J. R. Schwebke, A. Rompalo, S. Taylor et al., "Re-evaluating the treatment of nongonococcal urethritis: emphasizing emerging pathogens-a randomized clinical trial," Clinical Infectious Diseases, vol. 52, no. 2, pp. 163-170, 2011.

[4] J. Paavonen and W. Eggert-Kruse, "Chlamydia trachomatis: impact on human reproduction," Human Reproduction Update, vol. 5, no. 5, pp. 433-447, 1999.

[5] S. L. Gottlieb, R. C. Brunham, G. I. Byrne, D. H. Martin, F. $\mathrm{Xu}$, and S. M. Berman, "Introduction: the natural history and immunobiology of Chlamydia trachomatis genital infection and implications for Chlamydia control," Journal of Infectious Diseases, vol. 201, supplement 2, pp. S85-S87, 2010.

[6] S. D. Hillis, A. Nakashima, L. Amsterdam et al., "The impact of a comprehensive chlamydia prevention program in Wisconsin," Family Planning Perspectives, vol. 27, no. 3, pp. 108-111, 1995.

[7] C. Panchaud, S. Singh, D. Feivelson, and J. E. Darroch, "Sexually transmitted diseases among adolescents in developed countries," Family Planning Perspectives, vol. 32, no. 1, pp. 2445, 2000.

[8] F. Kamwendo, L. Forslin, L. Bodin, and D. Danielsson, "Decreasing incidences of gonorrhea- and Chlamydiaassociated acute pelvic inflammatory disease: a 25 -year study from an urban area of Central Sweden," Sexually Transmitted Diseases, vol. 23, no. 5, pp. 384-391, 1996.

[9] Centers for Disease Control and Prevention (CDC), "Chlamydia trachomatis genital infections-United States," Morbidity and Mortality Weekly Report, vol. 46, pp. 193-198, 1997.

[10] D. Fine, L. Dicker, D. Mosure, and S. Berman, "Increasing chlamydia positivity in women screened in family planning clinics: do we know why?" Sexually Transmitted Diseases, vol. 35, no. 1, pp. 47-52, 2008.

[11] R. C. Brunham, B. Pourbohloul, S. Mak, R. White, and M. L. Rekart, "The unexpected impact of a Chlamydia trachomatis infection control program on susceptibility to reinfection," Journal of Infectious Diseases, vol. 192, no. 10, pp. 1836-1844, 2005.

[12] Project SCREen, Review of chlamydia control activities in EU countries, Final report, 2011, http://www.ecdc.europa.eu/ en/publications/Publications/0805_TER_Review_of_Chlamydia_Control_Activities.pdf.
[13] Centers for Disease Control and Prevention, "Sexually transmitted disease surveillance 2007 supplement, chlamydia prevalence monitoring project annual report 2007," Tech. Rep., Department of Health and Human Services, Centers for Disease Control and Prevention, Atlanta, Ga, USA, January 2009.

[14] M. L. Rekart and R. C. Brunham, "Epidemiology of chlamydial infection: are we losing ground?" Sexually Transmitted Infections, vol. 84, no. 2, pp. 87-91, 2008.

[15] R. C. Brunham, B. Pourbohloul, S. Mak, R. White, and M. L. Rekart, "Reply to Hagdu and to Moss et al," Journal of Infectious Diseases, vol. 193, no. 9, pp. 1338-1339, 2006.

[16] N. J. Moss, K. Ahrens, C. K. Kent, and J. D. Klausner, "The decline in clinical sequelae of genital Chlamydia trachomatis infection supports current control strategies," Journal of Infectious Diseases, vol. 193, no. 9, pp. 1336-1338, 2006.

[17] I. J. Bakken and F. E. Skjeldestad, "Time trends in ectopic pregnancies in a Norwegian county 1970-2004-a populationbased study," Human Reproduction, vol. 21, no. 12, pp. 31323136, 2006.

[18] F. Kamwendo, L. Forslin, L. Bodin, and D. Danielsson, "Epidemiology of ectopic pregnancy during a 28 year period and the role of pelvic inflammatory disease," Sexually Transmitted Infections, vol. 76, no. 1, pp. 28-32, 2000.

[19] R. C. Brunham and M. L. Rekart, "Considerations on Chlamydia trachomatis disease expression," FEMS Immunology and Medical Microbiology, vol. 55, no. 2, pp. 162-166, 2009.

[20] H. Su, R. Morrison, R. Messer, W. Whitmire, S. Hughes, and H. D. Caldwell, "The effect of doxycycline treatment on the development of protective immunity in a murine model of chlamydial genital infection," Journal of Infectious Diseases, vol. 180, no. 4, pp. 1252-1258, 1999.

[21] S. A. Morré, A. J. van den Brule, L. Rozendaal et al., "The natural course of asymptomatic Chlamydia trachomatis infections: $45 \%$ Clearance and no development of clinical PID after one-year follow-up," International Journal of STD and AIDS, vol. 13, supplement 2, pp. 12-18, 2002.

[22] M. Molano, C. J. L. M. Meijer, E. Weiderpass et al., "The natural course of Chlamydia trachomatis infection in asymptomatic Colombian women: a 5-year follow-up study," Journal of Infectious Diseases, vol. 191, no. 6, pp. 907-916, 2005.

[23] E. Lyytikäinen, M. Kaasila, E. Hiltunen-Back et al., "A discrepancy of Chlamydia trachomatis incidence and prevalence trends in Finland 1983-2003," BMC Infectious Diseases, vol. 8, article 169, 2008.

[24] E. Lyytikäinen, M. Kaasila, P. Koskela et al., "Chlamydia trachomatis seroprevalence atlas of Finland 1983-2003," Sexually Transmitted Infections, vol. 84, no. 1, pp. 19-22, 2008.

[25] S. G. Morrison and R. P. Morrison, "A predominant role for antibody in acquired immunity to chlamydial genital tract reinfection," Journal of Immunology, vol. 175, no. 11, pp. 7536$7542,2005$.

[26] F. Kamwendo, L. Forslin, L. Bodin, and D. Danielsson, "Decreasing incidences of gonorrhea- and Chlamydiaassociated acute pelvic inflammatory disease: a 25-year study from an urban area of Central Sweden," Sexually Transmitted Diseases, vol. 23, no. 5, pp. 384-391, 1996.

[27] R. R. Ingalls, P. A. Rice, N. Qureshi, K. Takayama, G. S. Lin, and D. T. Golenbock, "The inflammatory cytokine response to Chlamydia trachomatis infection is endotoxin mediated," Infection and Immunity, vol. 63, no. 8, pp. 3125-3130, 1995.

[28] R. C. Brunham and M. L. Rekart, "The arrested immunity hypothesis and the epidemiology of chlamydia control," Sexually Transmitted Diseases, vol. 35, no. 1, pp. 53-54, 2008. 
[29] I. Miyairi, K. H. Ramsey, and D. L. Patton, "Duration of untreated Chlamydial genital infection and factors associated with clearance: review of animal studies," Journal of Infectious Diseases, vol. 201, supplement 2, pp. S96-S103, 2010.

[30] K. A. Fields and T. Hackstadt, "The chlamydial inclusion: escape from the endocytic pathway," Annual Review of Cell and Developmental Biology, vol. 18, pp. 221-245, 2002.

[31] G. Zhong, L. Liu, T. Fan, P. Fan, and H. Ji, "Degradation of transcription factor RFX5 during the inhibition of both constitutive and interferon $\gamma$-inducible major histocompatibility complex class I expression in chlamydia-infected cells," Journal of Experimental Medicine, vol. 191, no. 9, pp. 1525-1534, 2000.

[32] H. D. Caldwell, H. Wood, D. Crane et al., "Polymorphisms in Chlamydia trachomatis tryptophan synthase genes differentiate between genital and ocular isolates," Journal of Clinical Investigation, vol. 111, no. 11, pp. 1757-1769, 2003.

[33] S. D. Reighard, R. L. Sweet, C. V. Miguel et al., "Endometrial leukocyte subpopulations associated with Chlamydia trachomatis, Neisseria gonorrhoeae, and Trichomonas vaginalis genital tract infection," American Journal of Obstetrics and Gynecology, vol. 205, no. 4, pp. 324.e1-324.e7, 2011.

[34] A. L. Graham, J. E. Allen, and A. F. Read, "Evolutionary causes and consequences of immunopathology," Annual Review of Ecology, Evolution, and Systematics, vol. 36, pp. 373-397, 2005. 


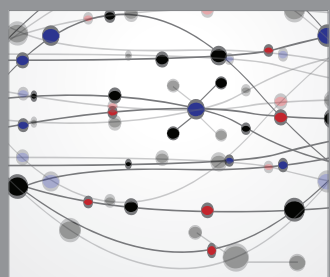

The Scientific World Journal
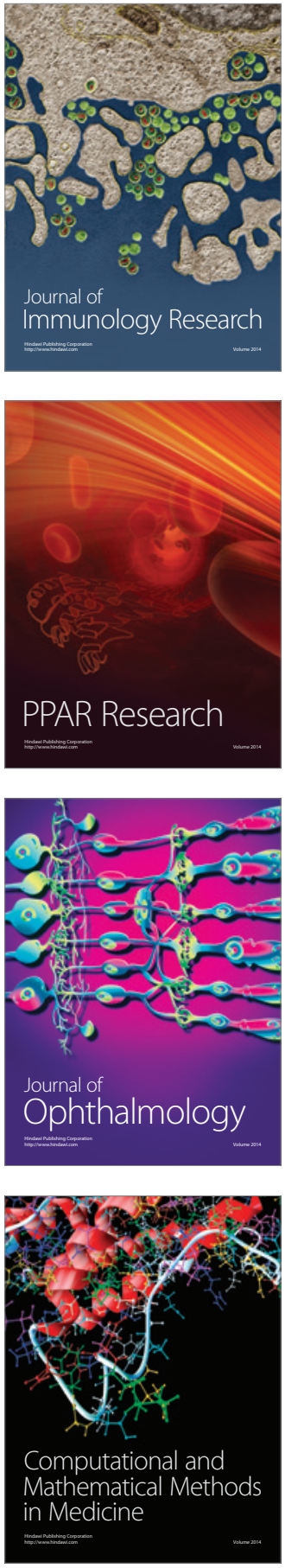

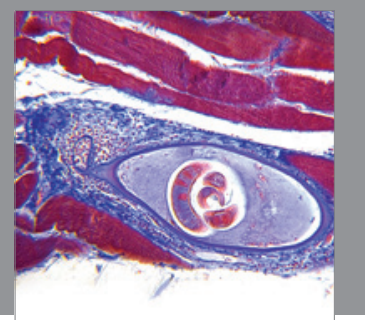

Gastroenterology

Research and Practice
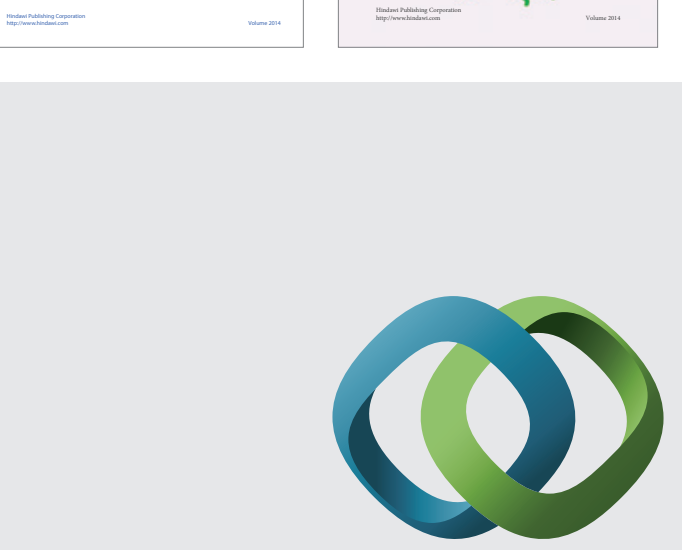

\section{Hindawi}

Submit your manuscripts at

http://www.hindawi.com
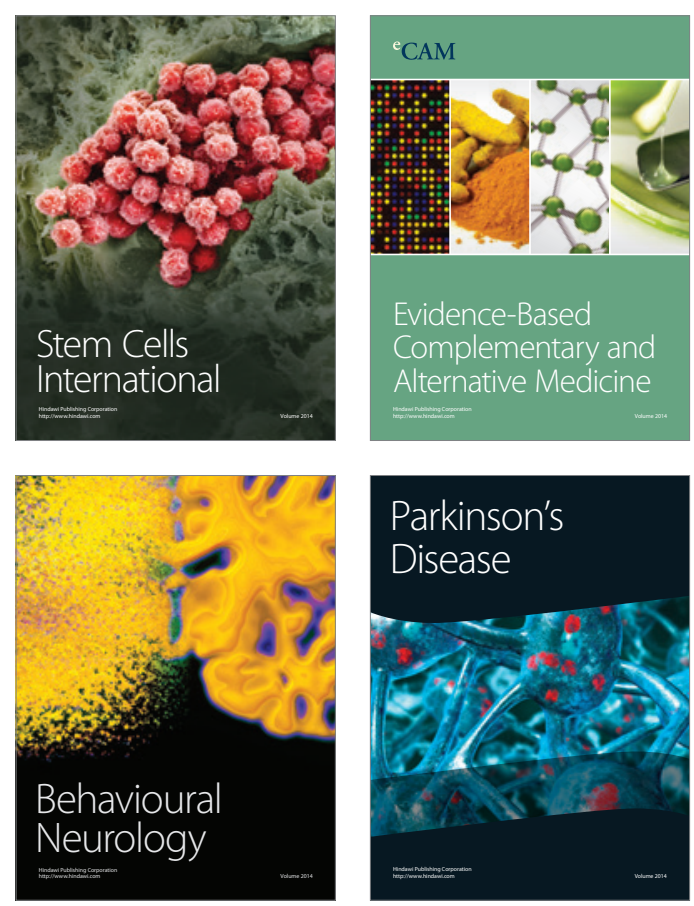

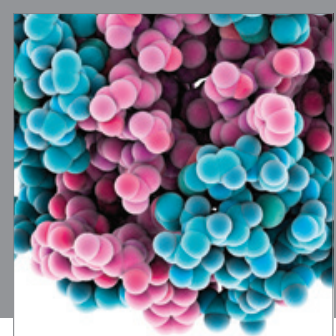

Journal of
Diabetes Research

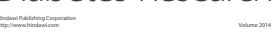

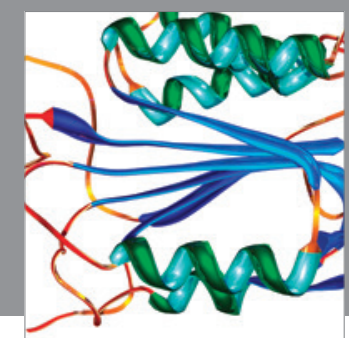

Disease Markers
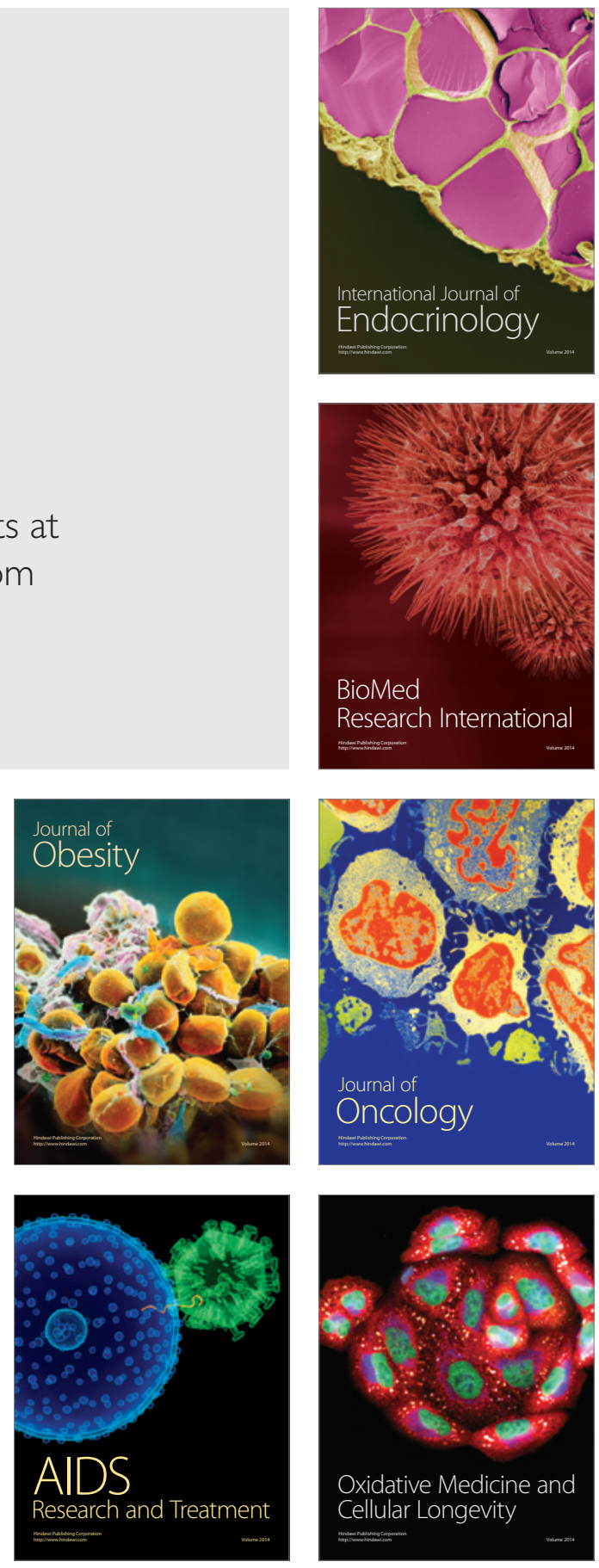